\title{
USE OF NATURALLY-OCCURRING ENZYME VARIATION TO DETECT AND MAP GENES CONTROLLING QUANTITATIVE TRAITS IN AN INTERSPECIFIC BACKCROSS OF TOMATO
}

\author{
STEVEN D. TANKSLEY,* HERCULANO MEDINA-FILHO $\dagger$ and CHARLES M. RICK \\ Department of Vegetable Crops, University of California, Davis 95616
}

Received 29.ix. 81

\section{SUMMARY}

A backcross between the inbred parents, Lycopersicon esculentum (LA 490, recurrent parent) and Solanum pennellii (LA 716) constitutes ideal material for an investigation of linkage between enzyme marker genes and loci determining quantitative traits (QTL): the chromosomes of the parents are the same in number and are homosequential; the parents differ for well-defined alleles at 12 enzymatic loci, which represent at least 8 of the 12 chromosomes, and large differences exist between the parents for measurable metric traits.

The four metric traits investigated in this study were inherited in typical quantitative fashion; the marker segregations fit monogenic ratios for all except four loci, for which there were deviations of three favoured alleles of the recurrent parent, in agreement with frequent observations in interspecific hybrids. Association between QTL and mapped enzyme markers were detected statistically from calculations based on the normal distribution. In this manner a minimum of 21 QTL were mapped.

Two pairs of linked enzyme markers permitted application of a three-point mapping procedure by comparing the data with models expected for various positions of QTL. Data for three QTL fitted predictions specified for a locus closer to one marker than the other, and data for one QTL met all criteria save one for a locus between the two markers.

Certain QTL coding for stigma exsertion and leaf ratio have effects opposite to those expected from parental values; i.e., for these traits both parents contribute alleles coding for positive and negative effects, resulting in the observed transgressive variation. When values observed for QTL linked with pairs of independent markers are compared with sums of separate values for each, significant epistatic interactions are detected. Although the extent of epistasis could not be determined precisely, it is probably not large. The paired interactions identified additional QTL whose individual effects were not otherwise detectable.

Enzymatic markers offer a number of advantages for the analysis of quantitative genetic variation which include: (a) lack of detectable effects on the morphology and physiology of the soma, (b) codominant expression, permitting exact identification of genotypes, and (c) lack of epistasis, allowing classification of any number of such markers segregating simultaneously. A tester stock with linked enzyme markers on each chromosome therefore could conceivably be utilized to analyze the total quantitative differences between two parents in a single segregating progeny. Such a tester stock might be especially useful for probing exotic germplasm for genes controlling quantitative characters of economic importance.

The methods of analysis outlined here could easily be adapted for analysis of $F_{2}$ populations or generations from other mating designs where a fuller array of genetic variation would be revealed.

Present addresses: * Department of Horticulture, New Mexico State Univ., Las Cruces, New Mexico 88003; † Instituto Agronômico, Campinas, Brazil. 


\section{INTRODUCTION}

SAX (1923) found an association of seed size (a quantitatively inherited character) with seed coat pigmentation (a discrete monogenic character). He attributed this correlation to linkage of seed size genetic factor(s) to the alleles for seed colour. A number of reports have since shown linkage of genetic factors controlling quantitative variation with discrete marker genes (for a review see Thompson and Thoday, 1979). The basic approach of using marker genes in segregations to detect genes controlling quantitative traits was put forth as a viable technique for systematically analyzing quantitative variation by Thoday (1961).

One problem inherent in this method of genetic analysis stems from the nature of the marker genes that one has classically been forced to use. Most of these markers are recessive, and when homozygous impart some morphological abnormality to the individual. The recessive-dominant allelic interaction also presents a problem. When using recessive marker alleles, the analysis is almost always confined to a backcross to the recessive homozygous tester stock. An $\mathrm{F}_{2}$ generally would yield considerably less information and a backcross to the dominant homozygous parent would yield no information since the marker gene segregation would not be detected.

A second problem with classical markers is the possibility of confounding effects of the marker genes with effects of the quantitative genetic factors being investigated. In some cases this problem has been overcome, albeit at the expense of considerable effort and time (Breese and Mather, 1957, 1960; Jayakar et al., 1977).

To make this technique of analyzing quantitative genetic variation with marker genes more powerful and easily applicable, it would be desirable to have a large number of marker genes distributed throughout the genome with alleles interacting in a codominant fashion. The alleles of these marker genes should produce little or no effect on the morphology of the organism in question, and each marker should be unequivocally identifiable even if a large number are segregating in the same cross. Enzyme marker genes fit these qualifications and, as indicated by our experience in Lycopersicon, are ideal candidates for such study.

In applying a test for using enzyme markers for quantitative genetic analysis, we crossed individuals from two morphologically distinct species of tomato which were homozygous for allelic differences at 12 enzymic loci. By monitoring, in a backcross population, the segregation of these loci-most of which have been mapped (see Tanksley and Rick, 1980)-we have been able to map a number of the genetic factors controlling several quantitatively inherited characters. Although our experiments were limited to the first backcross, where recessive factors and some of the epistatic variation would not be expressed, the approach to be outlined could well be applied in analyzing the range of genetic variation found in an $F_{2}$ population. The potential of enzyme markers to accelerate selection in this backcross is treated elsewhere (Tanksley et al., 1981).

\section{MATERIALS AND METHODS}

Hybrids were made between Lycopersicon esculentum cv. VF36 (LA 490) and the related wild species, Solanum pennellii (LA 716 from Atico, 
Peru). Both are highly inbred and homozygous for all tested loci. VF36 was used as the pistillate parent since unilateral compatibility precludes the reciprocal cross (Rick, 1960). Despite their allocation to different genera these two species are easy to hybridize, the $F_{1}$ 's experiencing nearly normal chromosome pairing and sufficiently high fertility to permit controlled introgression (Khush and Rick, 1963). Backcross seeds were obtained by using $F_{1}$ pollen in crosses to VF36.

\section{(i) Electrophoretic enzyme assays}

Five hundred $\mathrm{BC}_{1}$ seeds sown in wooden flats in the greenhouse produced approximately 400 viable seedlings. Six weeks later when they were approximately $15 \mathrm{~cm}$ tall, these plants were transplanted into a $1: 1$ mixture of sand and soil supplemented with fertilizer-a treatment that stimulates new root growth and thus high levels of enzyme activity. One week later the plants were assayed via starch gel electrophoresis to determine their individual genotypes with respect to the following segregating enzyme loci: Aps-1, Aps-2, Got-2, Prx-1, Prx-2, Prx-4, Prx-7, Est-4, Est-7, Pgm-2, $P g i-1$, and $S k d h-1$. Subsequently, these plants along with several replicates of the two parents and $F_{1}$ hybrid were transplanted in the field for measurement of quantitative traits. The mapped enzyme loci scored mark eight of the 12 chromosomes and two unmapped genes-Est-4 and Pgi-1-are known to be independent of the ten other enzymic genes-hence must mark significant additional parts of the genome, if not additional chromosomes. Methods of electrophoresis, enzyme extraction and map positions of the enzyme loci can be found in Tanksley and Rick (1980) and Tanksley (1981).

\section{(ii) Measurement of metric characters}

Measurements were taken on all individual plants of parents, $F_{1}$, and the backcross for the following quantitative traits: leaf ratio, stigma exsertion, fruit weight, and seed weight. Leaf ratio (width divided by length) differs between the orbicular segments of $S$. pennellii and the lanceolate ones of VF36. Stigma exsertion, measured from the mouth of the anther tube to the top of the stigma, varies from the exserted condition in $S$. pennellii to insertion in VF36. Means for these two characters were calculated from three independent measurements on each plant.

All plants were hand self-pollinated to assure adequate fruit set. Fruit weight was determined from a mean of six ripe fruits per plant, the minimum being two. A random sample of ten seeds from each plant was weighed to estimate the mean.

\section{RESUlTS AND DISCUSSION}

(i) Monogenic segregations

The segregation at each locus was tested against the expected 1:1 ratio. In the total population, four of the twelve enzyme markers yielded ratios that deviated significantly from expectation (table 2). Segregations of Aps-2 (chromosome 8), Skdh-1 (chromosome 1), and $\operatorname{Prx}-7$ (chromosome 3) showed a significant excess of $L$. esculentum homozygotes, whereas a significant excess of heterozygotes was observed for Prx-4 (chromosome 


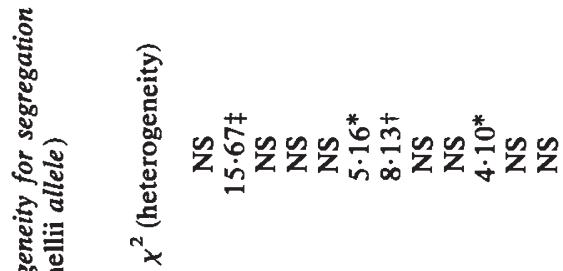

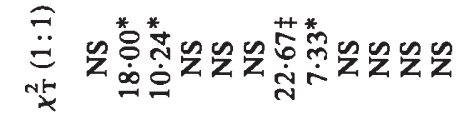

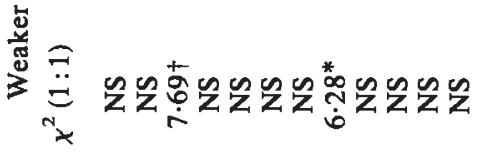

80

$\infty$

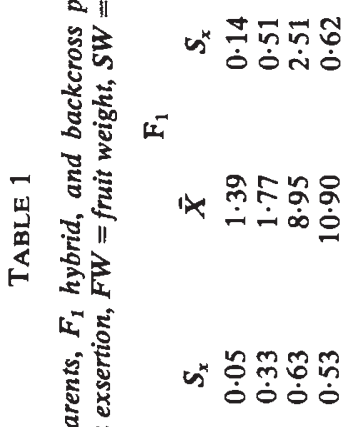

ธूँّั

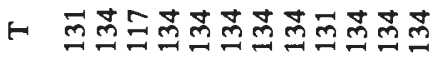

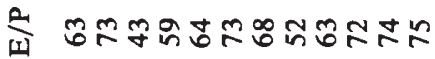

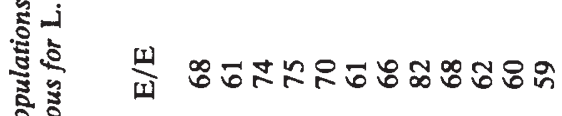

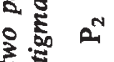

焉

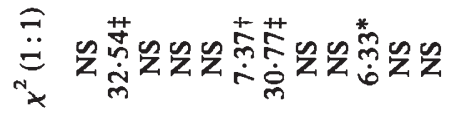

造范

ฟ ํํำ웜

है

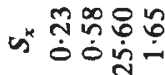

$$
\begin{aligned}
& =
\end{aligned}
$$

.

氙

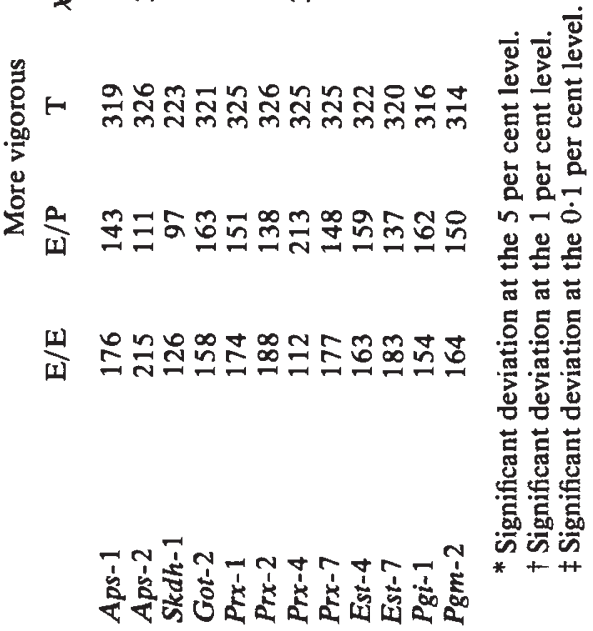

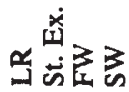



竎

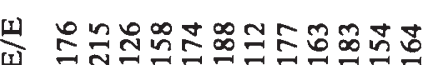

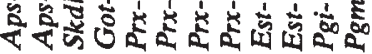


10). Our results are thus in general agreement with those of Rick (1969, 1972) for morphological markers on the three chromosomes, 3, 8, and 10. The latter investigations did not test chromosome 1.

In view of these distortions, each population was subdivided into stronger and weaker seedlings, the division being arbitrarily based on size of plant. The segregation data were compiled for each subgroup and Chi-square tests against $1: 1$ and for heterogeneity between subgroups calculated (table 2).

Two of the loci with overall distorted segregations showed differential segregation between subpopulations. For $A p s-2$ the more vigorous subpopulation deviated $2: 1, L$. esculentum homozygotes to heterozygotes, but segregation of the weaker group did not deviate significantly from $1: 1$. For the $\operatorname{Pr} x-4$ locus, the distortion was of the opposite type: the more vigorous individuals segregated $2: 1$ with an excess of heterozygotes, whereas the weaker group segregated approximately $1: 1$. In the segregations of Est-7 and Prx-2, an interesting reversal occurred in the direction of skewing between subpopulations. The more vigorous deviated significantly toward an excess of the recurrent parent alleles, whereas the weaker showed a slight, non-significant excess of heterozygotes. Curiously, the massed data of these two subpopulations did not deviate significantly from $1: 1$. Presumably the deviations of the weaker lot negated the significant distortions in the more vigorous.

Overall, seven significant deviations were encountered in the subpopulations and of these, six favoured the recurrent parent allele. In this respect the results are in general agreement with other tests of segregations in interspecific hybrids (Stephens, 1949; Rick, 1963, 1969, 1972). The fact that skewing at single loci differed significantly between the more vigorous and the weaker subpopulations for four of the twelve loci studied indicates that strong selection is acting at the seedling stage and can account for much of the overall skewness. Part of the single locus skewing may also be due to pollen competition during fertilization. The occurrence of this latter mechanism has been well documented (Zamir et al., 1981).

\section{(ii) Segregation of quantitative traits}

Information concerning the behaviour of the four quantitative traits in parents, $F_{1}$, and backcross generations is summarized in table 1 . Typical of quantitative characters in general, all exhibit continuous variation in the backcross. The genetic component of variation (broad sense heritability in comparisons between $F_{1}$ and $B C$ ) is relatively high for all except leaf ratio.

Leaf segments of $S$. pennellii are nearly circular in outline, having a mean leaf ratio (LR) of 0.96 , whereas those of VF36 are lanceolate and have a mean LR of 1.90. Means of the $F_{1}$ and $B C$ fall between the parental limits but demonstrate some degree of dominance of the wild parent.

In $S$, pennellii and the $F_{1}$, stigma exsertion is positive and nearly equal, revealing a high level of dominance of this parent, whereas the stigma is deeply inserted in the VF36 parent. The same degree of dominance is not revealed in $\mathrm{BC}$, the mean value falling very close to the midpoint between $P_{1}$ and $F_{1}$. Segregation in $B C$ is highly transgressive, greatly exceeding the limits of $P_{1}$ and $F_{1}$. 
Fruit of VF36 are 76 times heavier than those of $S$. pennellii. The mean fruit weights of the $\mathrm{F}_{1}$ and $\mathrm{BC}$ closely approximate the geometric means of the parental fruit weights in keeping with the observations in previous studies of the genetics of this character in tomatoes (MacArthur and Butler, 1938; Powers, 1942). The BC segregation is thus highly skewed toward the low parent. Dominance of small size is evident in the $F_{1}$ seed weight, but the $\mathrm{BC}$ mean very closely approximates the midpoint between $\mathrm{P}_{1}$ and $F_{1}$.

\section{(iii) Detection of linkage between enzyme loci and quantitative-trait loci}

Following the convention of Geldermann (1975) we shall refer to the segregating loci affecting the quantitative traits under study as quantitative trait loci (QTL). In order to detect linkage of enzyme loci with QTL controlling genetic variation of the traits-leaf ratio, stigma exsertion, fruit weight, and seed weight - the backcross data were divided into two groups with respect to each enzyme locus; one, all heterozygotes, the other all homozygotes. Differences between the means were calculated and twotailed significance levels computed for these differences based on the normal distribution:

$$
z_{\bar{d}}=\frac{\bar{x}_{1}-\bar{x}_{2}}{s_{\bar{d}}}
$$

(table 3). A significant difference in the means between homozygotes and heterozygotes was interpreted as linkage of QTL to the enzyme locus.

\section{(iv) Estimating the minimum number of QTL}

The minimum number of QTL controlling a quantitative character was taken to be the total number of independent enzyme loci demonstrating a significant effect on that character. Since some of the enzyme loci are linked (Skdh-1 and Prx-1,Prx-2 and Est-7, Est-4 and Pgi-1), only one of the linked pair was counted if both showed significance. This conservative approach seemed appropriate since the same QTL could be responsible for significant effects associated with both linked enzyme loci.

No attempt was made to estimate the magnitude of the effect a QTL must have on a character in order to be detected, as this depends on a number of factors including heritability, degree of additivity, size of the backcross population sampled, and proximity to an enzyme marker.

\section{(v) Test for single vs. multiple $Q T L$}

As already pointed out, more than one QTL can be linked to a given enzyme marker, the combined contributions causing the observed effect attributed to a marker. In certain instances there is a method of directly testing for single vs. multiple QTL associated with a given marker. This is restricted to QTL situated close to linked pairs of enzyme loci-in this case, $S k d h-1-P r x-1, \operatorname{Pr} x-2-E s t-7$, and Pgi-1-Est-4. The logic behind the test is as follows: If a detected "effect" showing association with a given marker is caused by multiple QTL, equally distributed with similar effects, then the genetic cause of the "effect" cannot, with the type of data generated 


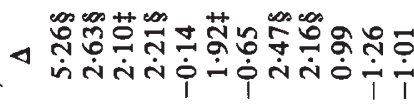

造

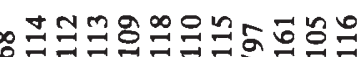

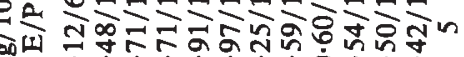
E 营 四 ช ڤ

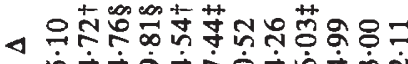

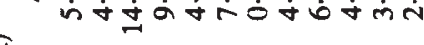
爱

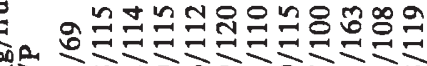

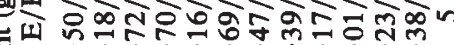

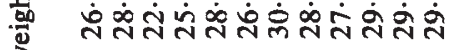

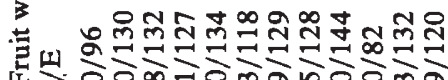

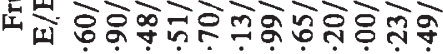

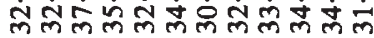

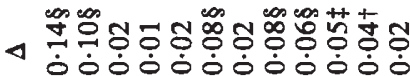

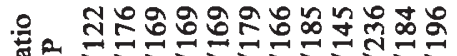

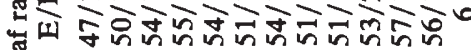

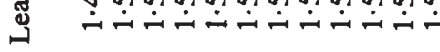
है

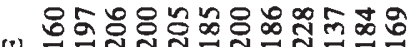

M

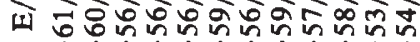

s.

$\infty$
$\infty$
$\infty$
$\infty$
$i$
$i$

돌

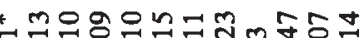

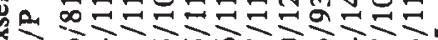

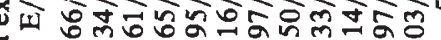

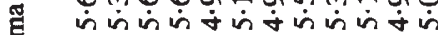

些

๒

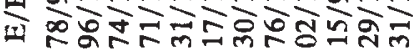

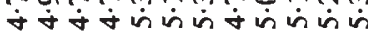

ป 
here, be mapped to a specific point on the chromosome. If, on the other hand, a single QTL or a tightly linked cluster of QTL causes the observed effect, then it should in theory be possible to associate the effect more strongly with one marker than the other. In some cases it might be possible to map the effect to a precise locus on the chromosome relative to the two linked markers.

\section{TABLE 4}

Models and submodels giving all possible gene locations ( $\alpha$ and $\beta=$ isozymic loci, $x=$ quantitative gene)

Model I quantitative gene closer to $\alpha$ than $\beta$

Model II quantitative gene closer to $\beta$ than $\alpha$

Model III quantitative gene equidistant from $\alpha$ and $\beta$ (between $\alpha$ and $\beta$ )

Submodel A quantitative gene between $\alpha$ and $\beta$

Submodel B quantitative gene not between $\alpha$ and $\beta$

Combinations of models and submodels

IA
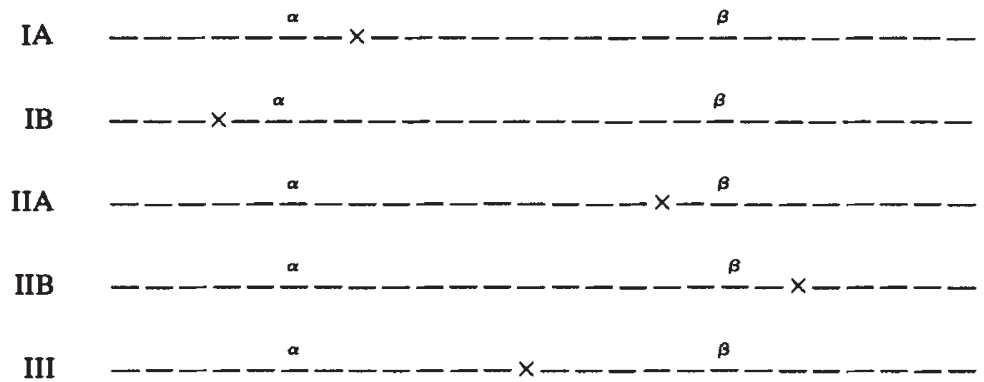

All of the possible positions of a single QTL with respect to two linked markers are given in table 4 . The possibilities are broken down into two considerations: (a) Is the QTL closer to one marker than the other (Models I-III)? (b) Is it between or to one side of the two linked markers (Submodels A, B)? If the backcross data are resolved into four classes representing all possible combinations of linked marker genotypes, the differences in the means between these classes can be used to answer these questions. Table

\section{TABLE 5}

Predicted differences between means of two linked loci combinations based on models I-III and submodels $A, B$ (+= positive value; $-=$ negative value; $O=$ no difference; $N P=$ no prediction; $A=E / E E / E, B=E / E E / P, C=E / P E / E, D=E / P E / P)$

\begin{tabular}{|c|c|c|c|c|c|c|c|c|c|c|c|c|c|}
\hline & & & Mo & del & & & & & Sul & del & & & \\
\hline & & & & & & & & & & & & & \\
\hline & 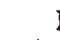 & I & I & $I$ & III & & & & & & 1 & & \\
\hline & $\Delta^{*}$ & $\Delta \dagger$ & $\Delta^{*}$ & $\Delta \dagger$ & $\Delta^{*} \Delta \dagger$ & $\Delta^{*}$ & $\Delta \dagger$ & $\Delta^{*}$ & $\Delta \dagger$ & $\Delta^{*}$ & $\Delta \dagger$ & $\Delta^{*}$ & $\Delta \dagger$ \\
\hline B-C & + & - & - & + & 0 & + & - & - & + & + & - & - & + \\
\hline A-B & NP & NP & + & - & 0 & + & - & + & - & 0 & 0 & + & - \\
\hline C-D & NP & NP & + & - & 0 & + & - & + & - & 0 & 0 & + & - \\
\hline$A-C$ & + & - & NP & NP & 0 & + & - & - & + & + & - & 0 & 0 \\
\hline B-D & + & - & NP & NP & 0 & + & - & - & + & + & - & 0 & 0 \\
\hline
\end{tabular}

* Recurrent parent has larger value for quantitative trait compared to $\mathrm{F}_{1}$ (leaf ratio, fruit weight, seed weight).

$\dagger$ Recurrent parent has small value for quantitative trait compared to $F_{1}$ (stigma exsertion). 
5 lists the predicted direction $(+$ or -$)$ that these differences should take for different locations of a single QTL (different combinations of Models I-III and submodels A, B).

A breakdown of the data for linked enzyme markers showing significant effects on different quantitative characters is given in table 6 . The statistical significance of the differences was tested using the normal distribution:

$$
Z=\frac{\bar{x}_{1}-\bar{x}_{2}}{\sqrt{\frac{\mathrm{MSE}}{n_{1}}+\frac{\mathrm{MSE}}{n_{2}}}} .
$$

The MSE (mean square error) was obtained from a series of 2-way analyses of variance using the linked loci as treatments. By matching the significant differences between the means with the predictions of the models in table 5, those "effects" that behave like single QTL can be mapped. Those cases for which no significant difference in the means was observed are candidates for multiple, recombining QTL.

\section{(vi) Detected QTL}

At least five QTL were detected for each of the traits studied, with the average being $5 \cdot 2$ (table 3 ). Six of the detected QTL were associated with linked pairs of enzyme markers, thereby permitting application of the test outlined above of single vs. multiple QTL. Three detected QTL fit models I or II from table 5, indicating that they are positioned closer to one marker than the other and suggesting that they might be single QTL. None of the detected QTL fits perfectly any submodel which would allow positioning between or to one side of the markers. However, one of the QTL for leaf ratio (Skdh-1-Prx-1) did fit the between-locus case (Model I, submodel A) for all but one of the predicted differences, indicating that it might reside between these two enzyme marker loci which themselves are $17 \mathrm{~cm}$ apart.

\section{(vii) Plus and minus effects of QTL}

By comparing values of the two parents and the $F_{1}$ hybrid one might predict whether the QTL contributed by each parent will have a positive or negative effect. For example, VF36 has fruit approximately 100 times heavier than $S$. pennellii. Thus the QTL contributed by VF36 should cause an increase in fruit weight. These predictions were met by all the QTL detected for fruit weight and seed weight. However, for the other two characters (stigma exsertion and leaf ratio), some QTL were detected which produced effects opposite to these predicted by the parental values. Thus one of the leaf ratio QTL contributed by $S$. pennellii caused a slight but significant increase in this character in $\mathrm{BC}_{1}$ plants even though $S$. pennellii has a smaller leaf ratio value (table 3 ). For stigma exsertion the results were even more striking. Two of the five QTL detected, caused effects in the direction opposite to those predicted by the parents (table 3 ). Based on these observations, leaf ratio and stigma exsertion might be characters for which transgressive variation could be recovered in segregating generations. By combining a large number of positive or negative QTL contributed by both parents, some backcross individuals might exceed the 


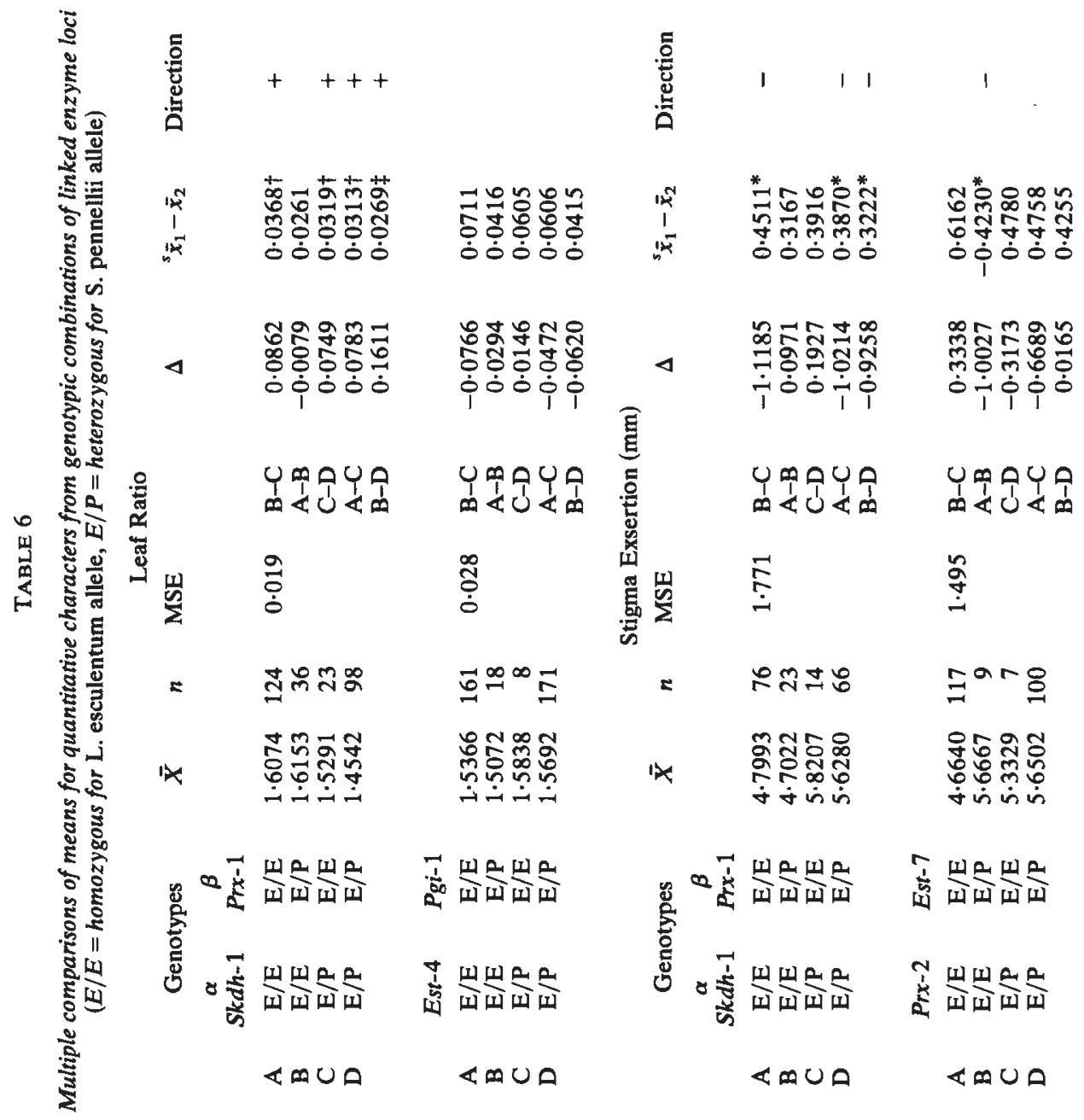


氖 +

冚

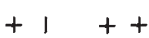

in

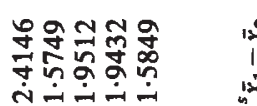

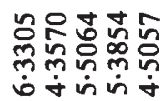

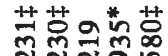
సิํํํํำ 禹战它

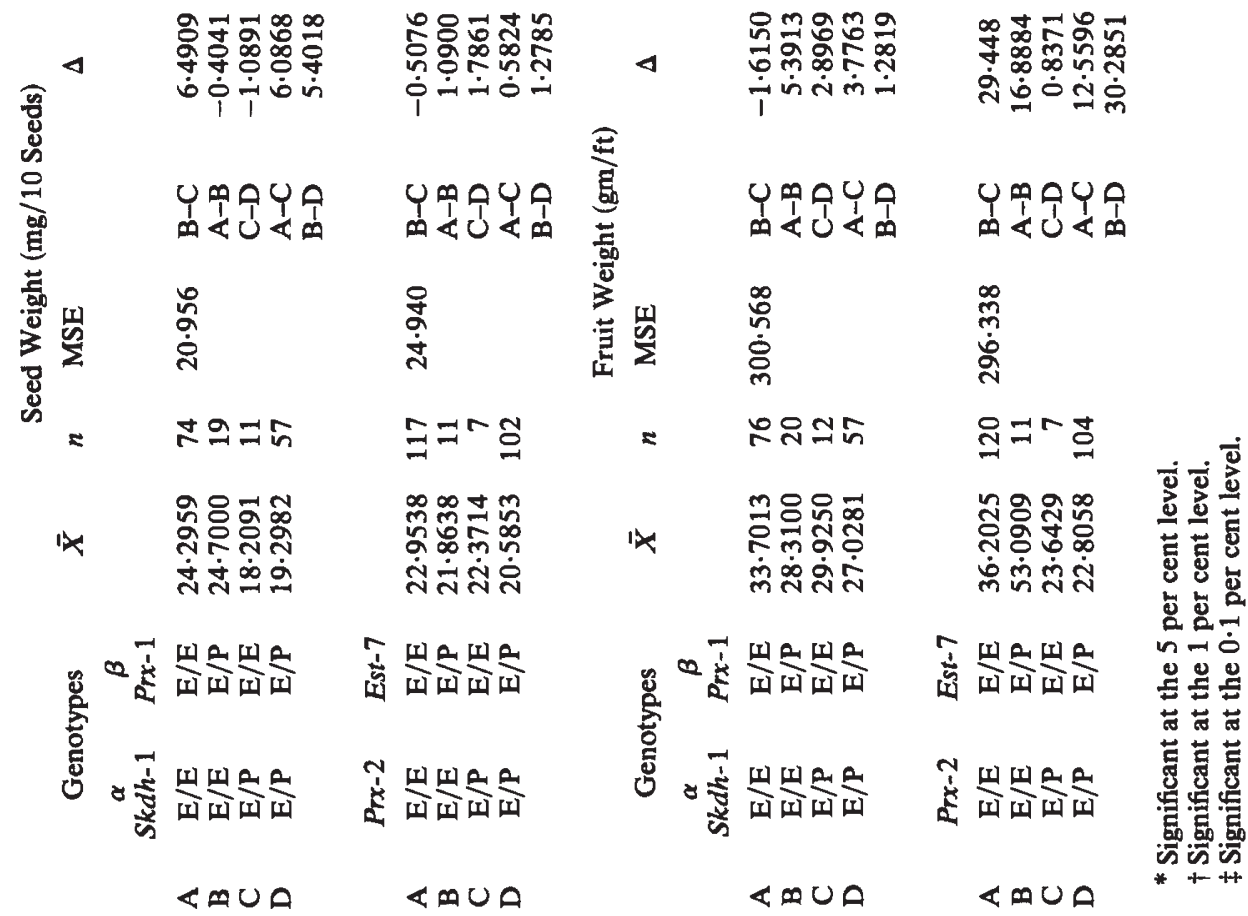


parental extremes. This was clearly the case for stigma exsertion where some individuals had stigmas exserted or inserted to a degree greatly exceeding either parent (table 1).

\section{(viii) Test for epistatic interaction between $Q T L$}

Interacting effects of detected QTL on the four metric characters were tested by 2 -way analysis of variance in which pairs of marker loci served as treatments. Significant interaction terms from AOV were interpreted as indications of epistatic interaction between QTL and were observed for all four traits. Amongst the total of 274 possible comparisons for two-locus interactions, 18 showed significant interactions, 13 at a 5 per cent probability level, five at the 1 per cent level (table 7). In such a large number of traits, obviously not all comparisons with associated low probabilities can indicate real differences. Yet, in terms of the 1 per cent group alone, the total amount of interaction between loci is clearly more than expected by random deviation alone. Furthermore, of the seven interactions affecting seed weight, four are with QTL linked with $P g m-2$, the remainder, with Aps-1. This delimitation, significant at $p=(21 / 66)^{6}=0 \cdot 00104$, suggests that the activity of seed weight QTL linked with Aps-1 and Pgm-2 is contingent upon the presence or absence of other segregating QTL. In another situation, an unexpected type of interaction occurred for the effect

\section{TABLE 7}

Means for quantitative characters based on breakdown of genotypes for loci showing epistatic interaction. Pairs of numbers indicate genotypes $(1=$ homozygous for $\mathrm{L}$. esculentum allele; $2=$ heterozygous for $\mathrm{S}$. pennellii allele)

\begin{tabular}{|c|c|c|c|c|}
\hline Leaf ratio & 1,1 & 1,2 & 2,1 & 2,2 \\
\hline 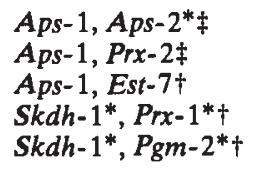 & $\begin{array}{l}1.572 \\
1.533 \\
1.574 \\
1.607 \\
1.660\end{array}$ & $\begin{array}{l}1.536 \\
1.533 \\
1.542 \\
1.615 \\
1.556\end{array}$ & $\begin{array}{l}1.578 \\
1.536 \\
1.534 \\
1.529 \\
1.494\end{array}$ & $\begin{array}{l}1.485 \\
1.554 \\
1.555 \\
1.454 \\
1.438\end{array}$ \\
\hline \multicolumn{5}{|c|}{ Stigma exsertion $(\mathrm{mm})$} \\
\hline 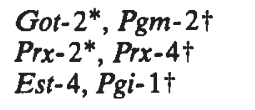 & $\begin{array}{l}4 \cdot 723 \\
4.911 \\
5.285\end{array}$ & $\begin{array}{l}4.922 \\
4.623 \\
5 \cdot 333\end{array}$ & $\begin{array}{l}5 \cdot 717 \\
5 \cdot 455 \\
5 \cdot 695\end{array}$ & $\begin{array}{l}5 \cdot 346 \\
5 \cdot 707 \\
4 \cdot 985\end{array}$ \\
\hline \multicolumn{5}{|l|}{ Fruit weight (g/fruit) } \\
\hline 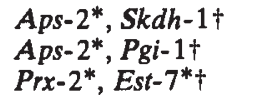 & $\begin{array}{l}32 \cdot 814 \\
33 \cdot 273 \\
36 \cdot 203\end{array}$ & $\begin{array}{l}30 \cdot 670 \\
32 \cdot 581 \\
53 \cdot 091\end{array}$ & $\begin{array}{l}32 \cdot 106 \\
29 \cdot 304 \\
23 \cdot 643\end{array}$ & $\begin{array}{l}23 \cdot 903 \\
24 \cdot 565 \\
22 \cdot 806\end{array}$ \\
\hline \multicolumn{5}{|c|}{ Seed weight (mg/10 seeds) } \\
\hline 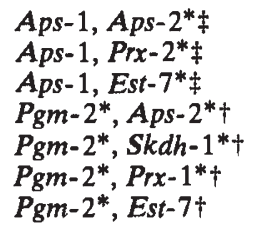 & $\begin{array}{l}21 \cdot 947 \\
23 \cdot 292 \\
23 \cdot 513 \\
24 \cdot 250 \\
25 \cdot 656 \\
24 \cdot 793 \\
24 \cdot 650\end{array}$ & $\begin{array}{l}21 \cdot 106 \\
20 \cdot 016 \\
19 \cdot 981 \\
21 \cdot 080 \\
19 \cdot 534 \\
21 \cdot 280 \\
23 \cdot 141\end{array}$ & $\begin{array}{l}23 \cdot 797 \\
22 \cdot 505 \\
22 \cdot 558 \\
21 \cdot 409 \\
23 \cdot 137 \\
21 \cdot 934 \\
21 \cdot 662\end{array}$ & $\begin{array}{l}19 \cdot 950 \\
21 \cdot 907 \\
21 \cdot 772 \\
20 \cdot 348 \\
19 \cdot 009 \\
19 \cdot 714 \\
20 \cdot 340\end{array}$ \\
\hline
\end{tabular}

* Single locus effect significant at 5 per cent level.

$\dagger$ Interaction significant at 5 per cent level.

$\ddagger$ Interaction significant at 1 per cent level. 
of the two linked markers $\operatorname{Pr} x-2$ and Est 7 with respect to fruit weight. The data in table 6 suggest that QTL linked to these two markers has a positive effect on fruit weight and that this QTL maps closer to $\operatorname{Prx}-2$ than to Est-7. The breakdown of the two-locus groups in table 7 indicates a much larger effect in the presence of the Est-7 S. pennellii allele. In other words, the recombinant chromosome produces the greatest boost in fruit weight. Otherwise no clear patterns are discernible in the data.

The dilemma of determining which of the interactions with $P \leqq 0.05$ are real cannot be resolved with our data. At any rate, the highly significant interactions and the aforementioned indications of nonrandomness reveal the occurrence of some epistatic interaction.

Twelve of the 18 interactions included one or both markers which singly did not significantly affect the characters in question; all five of the highly significant interactions belong in this group. The relationship suggests that a number of the loci affecting the quantitative traits were not detected by single-locus analysis. It seems likely that these undetected QTL are highly epistatic, possibly producing both positive and negative effects on the character, depending on the allelic composition at other loci throughout the genome. If such were the case, they would probably not be detected by the test for differences between homozygous and heterozygous marker loci since they produce no consistent effect. The procedures for detecting QTL are therefore undoubtedly biased toward loci with a higher degree of additivity.

\section{(ix) Advantages of enzyme markers for investigating quantitative traits}

Many of the advantages of enzyme markers over morphological markers for genetic studies have been discussed previously (Brown and Allard, 1969; Nielsen and Scandalios, 1974; Tanksley and Rick, 1980). In contrast to the usual recessive/dominance interaction of morphological markers, most enzyme alleles interact in a codominant manner, in which $\mathrm{F}_{2}$ segregations and backcrosses to either parent can be studied with comparable efficiency. Segregation of recessive morphological markers, on the other hand, can be detected in the backcross to only one parent, and in the $F_{2}$, far less information is yielded by a $3: 1$ than by the $1: 2: 1$ ratios typical of allozymes.

Even if a large number of enzyme loci segregate simultaneously in the same progeny, the genotype for each locus can be determined unequivocally in all individuals. It is therefore theoretically possible to use several enzyme markers on every chromosome. If a backcross or $F_{2}$ were segregating for such an array of markers, a very clear picture of the genetic architecture of the investigated quantitative trait would be likely to emerge. Theoretically all of the QTL of major consequence affecting a trait could be mapped and characterized with data from a single segregating progeny. Since the contribution of the entire genome is observed simultaneously, tests for epistatic interactions between QTL would be straightforward. The results presented here as well as those of many other researchers (e.g., Spickett and Thoday, 1966; Fasoulas and Allard, 1962) reveal that interactions between discrete QTL are common. In contrast, it is obviously difficult to establish the extent and nature of such interactions if only a few marker genes are segregating simultaneously-the usual situation with 
morphological markers. Likewise, many epistatic interactions would not be detected by chromosome substitution techniques, in which the action of only a small portion of the genome is studied at a time.

For wide crosses, as in the present example, in which the desired enzyme variation is already present, no new markers need to be introduced. Thus, the time required in terms of number of generations and crosses is drastically reduced and the difficulties accompanying the introduction of extraneous genetic variation is avoided. Nevertheless, for the procedure to be more generally applicable, it might be possible to introduce into a single stock a series of rare enzyme alleles. This stock, which would differ at all relevant enzyme marker loci with respect to most other conspecific lines, could be used to test for any QTL-controlled trait. If enough allele differences were obtained, it should be possible in a single cross to test 90-100 per cent of the genome for QTL. In tomato, 20 enzymic genes have been mapped and another 10 should be located in the near future, so that a more complete coverage can be expected. For virtually all of these loci, rare alleles are available for incorporation into a single stock.

Up to the present time, the mapping of QTL has been mainly limited to Drosophila for which the generation time is relatively short and a good assortment of genetic markers is available. By decreasing the number of crosses and generations required to map the QTL of various quantitative traits, the use of enzyme markers in quantitative genetic studies might be extended to other organisms.

Finally, in breeding programmes in both animals and plants, the identification and mapping of major genes controlling important quantitative traits offer certain possibilities. Such genes found to be closely linked with one enzyme marker (better if situated between two enzyme markers) could be transferred with great efficiency and certainty. The codominant enzyme alleles could be selected in early development stages, deferring direct selection for the quantitative character in question until advanced generations. It might be especially useful for probing exotic germplasm for important genes and transferring them into desirable backgrounds. Some aspects of this method of quantitative gene transfer have been considered by Soller and Plotkin-Hazen (1977).

Tomato is an ideal model system for quantitative genetic studies using enzyme markers. In addition to the advanced status of enzyme genetic studies in this plant, there are a number of related, crossable species which possess characteristics of economic importance including drought tolerance, salt tolerance and low temperature tolerance (Rick, 1973, 1975, 1978; Vallejos, 1979; Epstein et al., 1980). Presently there is great interest in transferring these traits into the cultivated tomato. Enzyme techniques as described in this paper might be used to study their inheritance and at the same time to facilitate their transfer into useful breeding lines.

Acknowledgments.-This research was supported in part by U.S. National Science Foundation Grant DEB-8005542. We are also grateful for the assistance of Anne Rundstrom, Maureen T. Farrell, and Dora G. Hunt in various phases of the project.

\section{REFERENCES}

BREESE, E. L., AND MATHER, K. 1957. The organization of polygenic activity within a chromosome in Drosophila. I. Hair characters. Heredity, 11, 373-395. 
BREESE, E. L., AND MATHER, K. 1960. The organization of polygenic activity within a chromosome in Drosophila. II. Viability. Heredity, 14, 375-400.

BROWN, A. H. D., AND ALLARD, R. W. 1969. Inheritance of isozyme differences among the inbred parents of a reciprocal recurrent selection population of maize. Crop Science, 9, $72-75$.

EPSTEIN, E., NORLYN, J. D., RUSH, D. W., KINGSBURY, R. W., KELLEY, D. B., CUNNINGHAM, G. A., AND WRONA, A. F. 1980. Saline culture of crops: a genetic approach. Science, 210, 399-404.

FASOULAS, A. C., AND ALLARD, R. W. 1962. Nonallelic gene interactions in the inheritance of quantitative characters of barley. Genetics, 47, 899-907.

GELDERMANN, H. 1975. Investigations on inheritance of quantitative characters in animals by gene markers. I. methods. Theor. Appl. Genet., 46, 319-330.

JAYAKAR, S. D., DELLA CROCE, L., SCACCHI, M., AND GUAZZOTTI, G. 1977. A genetic linkage study of a quantitative traits in Drosophila melanogaster. In E. Pollak, O. Kempthorne, T. B. Bailey, Jr. (eds.) Proceedings of the International Conference of Quantitative Genetics. The Iowa State Univ. Press.

KHUSH, G. S., AND RICK, C. M. 1963. Meiosis in hybrids between Lycopersicon esculentum and Solanum pennellii. Genetica, 33, 167-183.

MACARTHUR, J. W., AND BUTLER, L. 1938. Size inheritance and geometric growth processes in the tomato. Genetics, 23, 253-268.

NIELSEN, G., AND SCANDALIOS, J. G. 1974. Chromosomal location by use of trisomics and new alleles of an endopeptidase in Zea mays. Genetics, 77, 679-686.

POWERS, L. 1942. The nature of the series of environmental variances and the estimation of the genetic variances and the geometric means in crosses involving species of Lycopersicon. Genetics, 27, 561-575.

RICK, C. M. 1960. Hybridization between Lycopersicon esculentum and Solanum pennellii: phylogenetic and cytogenetic significance. Proc. Natl. Acad. Sci., 45, 78-83.

RICK, C. M. 1963. Differential zygotic lethality in a tomato species hybrid. Genetics, 48 , 1497-1507.

RICK, C. M. 1969. Controlled introgression of chromosomes of Solanum pennellii into Lycopersicon esculentum: segregation and recombination. Genetics, 62, 753-768.

RICK, C. M. 1972. Further studies on segregation and recombination in backcross derivatives of a tomato species hybrid. Biol. Zbl., 91, 209-220.

RICK, C. M. 1973. Potential genetic resources in tomato species: clues from observations in native habitats. In A. M. Srb (ed.) Genes, Enzymes, and Populations, Plenum Press, New York.

RICK, C. M. 1975. The tomato. In R. C. King (ed.) Handbook of Genetics, Vol. 2, Plenum Press, New York.

RICK, C. M. 1978. The tomato. Sci. Amer., 239, (2), 76.

SAX, K. 1923. The association of size differences with seed-coat pattern and pigmentation in Phaseolus vulgaris. Genetics, 8, 552-560.

SOLLER, M., AND PLOTKIN-HAZAN, J. 1977. The use of marker alleles for the introgression of linked quantitative alleles. Theor. Appl. Genet., 51, 133-137.

SPICKETT, S. G., AND THODAY, J. M. 1966. Regular responses to selections 3. Interaction between located polygenes. Genet. Res., Camb., 7, 96-121.

STEPHENS, S. G. 1949. The cytogenetics of speciation in Gossypium. I. selective elimination of the donor parent genotype in interspecific backcrosses. Genetics, 34, 627-637.

TANKSLEY, S. D. 1981. Map positions of $P r x-1$, Skdh-1, and Pgm-1. Rep. Tomato Genet. Coop. No. 31, 18.

TANKSLEY, S. D., AND RICK, C. M. 1980. Isozymic gene linkage map of the tomato: applications in genetics and breeding. Theor. Appl. Genet., 57, 161-170.

TANKSLEY, S. D., MEDINA-FILHO, H., AND RICK, C. M. 1981. The effect of isozyme selection on metric characters in an interspecific backcross of tomato-basis of an early screening procedure. Theor. Appl. Genet., 60, 291-296

THODAY, J. M. 1961. Location of polygenes. Nature, 191, 368-370.

THOMPSON, J. N., JR., AND THODAY, J. M. 1979. Ed. Quantitative Genetic Variation. Academic Press, New York.

VALLEJOS, C. E. 1979. Genetic diversity of plants for response to low temperature and its potential use in crop plants. In Lyons, J. M., D. Graham, and J. K. Raison (eds.) Low Temperature Stress in Crop Plants, Academic Press, New York.

ZAMIR, D., TANKSLEY, S. D., AND JONES, R. A. 1981. Haploid selection for low temperature tolerance of tomato pollen. (submitted). 